\title{
Maladie de Pompe à début tardif : une nouvelle molécule en enzymothérapie substitutive
}

\section{Françoise Chapon}

Résumé

Cet article [1] rapporte l'évaluation d'un nouveau traitement substitutif dans la maladie de Pompe par alpha avalglucosidase (ou néoGAA). Les patients inclus sont atteints de la forme tardive et ont bénéficié d'une enzymothérapie substitutive préalable par GAA, ou non (patients naïfs). Entre 2013 et 2015, répartis dans 17 centres, 24 patientsâgés de plus de 18 ans, ambulatoires et sans assistance respiratoire (capacité vitale - CV - $\geq 50 \%$ ), ont été inclus. Neufs patients naïfs sur 10 et $12 / 14$ préalablement traités par alglucosidase sur une durée de 9 mois ou plus, sont allés au bout de l'étude. Des perfusions aux doses de 5,10 ou $20 \mathrm{mg} / \mathrm{kg}$ ont été administrées une semaine sur deux sur une période de 24 semaines. La durée totale de l'étude pour chaque patient a été en moyenne de 41 semaines. L'évaluation a comporté l'appréciation des fonctions respiratoire et motrice, des données pharmacocinétiques (concentration plasmatique de l'enzyme avant, pendant, et après la perfusion - jusqu' 48 heures postperfusion -, visites réalisées sur les semaines 1,13 et 25), des données pharmacodynamiques (taux du glycogène dans le muscle squelettique évalué par résonance magnétique, du tétraglucose urinaire, contenu glycogénique dans la biopsie musculaire évalué par analyse automatisée en microscopie optique), des données de surveillance (réactions aux perfusions, effets indésirables, recherche d'anticorps anti-néoGAA, neutralisants, et anti-GAA). Le traitement a été globalement bien toléré à toutes les doses. Un seul patient, du groupe naiif, a présenté un effet indésirable sévère (détresse respiratoire et gêne thoracique) à la $9^{e}$ perfusion, à l'origine d'une sortie de l'étude. L'état clinique des patients est resté stable ou s'est amélioré. La concentration du produit a diminué de façon mono-exponentielle sans variation du profil sur les différentes semaines étudiées et les paramètres étaient sensiblement identiques dans les deux groupes de patients. Enfin, le taux de glycogène intramusculaire est resté stable.

\section{Commentaire}

La maladie de Pompe ou glycogénose de type 2 est liée à un déficit en alpha glucosidase acide (GAA).
Différentes formes sont décrites selon l'âge de survenue des symptômes, la forme la plus fréquente étant la forme tardive, caractérisée par l'atteinte des muscles proximaux des membres et des muscles respiratoires. Dans cette forme tardive, un traitement substitutif par enzyme recombinante, l'alglucosidase (Myozyme $^{\mathrm{R}}$, AMM en 2006) a permis une stabilisation de l'atteinte respiratoire et une amélioration des capacités de marche. Cependant, l'alglucosidase est plus efficace sur la surcharge glycogénique du muscle cardiaque que sur celle du muscle squelettique. Cela a été corrélé à une différence tissulaire dans l'expression du récepteur mannose 6-phosphate cation-indépendant (CIM6P)/insulin like growth factor-2 ou facteur de croissance-2 analogue à l'insuline) (IGF2) et dans la captation enzymatique. Ainsi la molécule utilisée dans l'étude ici rapportée, le néoGAA ou avalglucosidase est un recombinant de seconde génération comportant un taux plus important de M6P (sites de glycosylation) afin d'augmenter la captation médiée par le récepteur. Les travaux précliniques effectués dans le modèle murin de maladie de Pompe ont montré une affinité de l'avalglucosidase pour ces récepteurs MP6 1000 fois supérieure comparée à de celle de l'alglucosidase. Cette étude de phase I s'attache à l'évaluation de l'innocuité du traitement mais l'évaluation de l'efficacité n'est désignée qu' " exploratoire " du fait de la très petite cohorte. Les résultats montrant une bonne tolérance du produit encouragent à d'autres études sur un plus grand nombre de patients et une période plus longue afin de déterminer l'efficacité de l'avalglucosidase et de permettre des comparaisons avec celle de l'alglucosidase. Une étude en cours, COMET (NCT02782741), portant sur 96 patients traités soit par Myozyme ${ }^{\mathrm{R}}$ soit par néoGAA, doit se poursuivre jusqu'en 2020 et permettra de répondre à cette question.

Late-onset Pompe disease: a new molecule for enzyme replacement therapy

\section{LIENS D'INTÉRÊT}

L'auteur déclare n'avoir aucun lien d'intérêt concernant les données publiées dans cet article.

\footnotetext{
Françoise Chapon

$\mathrm{CHU}$

de Caen-Normandie,

Centre de Compétences des Maladies

Neuromusculaires,

14033 Caen, France

Contact

chapon-f@chu-caen.fr
}

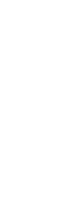

\footnotetext{
RÉFÉRENCES

1. Pena LDM, Barohn RJ, Byrne BJ, et al. Safety, tolerability, pharmacokinetics, pharmacodynamics, and exploratory efficacy of the novel enzyme replacement therapy avalglucosidase alfa (neoGAA) in treatment-naïve and alglucosidase alfa-treated patients with lateonset Pompe disease: a phase 1, open-label, multicenter, multinational, ascending dose study. Neuromuscul Disord $2019 ; 29: 167-86$.
} 